beams with different frequencies can be used, then we will have the possibility of seeing multicolour coherent superposition of vortex states, forming time-dependent superposition patterns such as 'Ferris wheels' seen in optical vortex beams ${ }^{12}$. More complex light-plasmon interaction even allows for the temporal control of the electron beams with attosecond precision ${ }^{3}$. The work opens up the new field of fourdimensional shaping of structured matter waves.
Jun Yuan

Department of Physics, University of York, York, UK. e-mail:jun.yuan@york.ac.uk

Published online: 6 May 2019

https://doi.org/10.1038/s41563-019-0375-7

References

1. Lloyd, S. M., Babiker, M., Thirunavukkarasu, G. \& Yuan, J. Rev. Mod. Phys. 89, 035004 (2017).

2. Béché, A., Van Boxem, R., Van Tendeloo, G. \& Verbeeck, J. Nat. Phys. 10, 26-29 (2013).

$\square \quad 3$. Priebe, K. E. et al. Nat. Photon. 11, 793-797 (2017).
4. Vanacore, G. M. et al. Nat. Commun. 9, 2694 (2018).

5. Vanacore, G. M. et al. Nat. Mater. https://doi.org/10.1038/s41563 019-0336-1 (2019)

6. Gorodetski, Y., Niv, A., Kleiner, V. \& Hasman, E. Phys. Rev. Lett. 101, 043903 (2008)

7. Barnes, W. L., Dereux, A. \& Ebbesen, T. W. Nature 424, 824-30 (2003).

8. Cho, S.-W., Park, J., Lee, S.-Y., Kim, H. \& Lee, B. Opt. Express 20, 10083-10094 (2012).

9. Asenjo-Garcia, A. \& García de Abajo, F. J. Phys. Rev. Lett. 113, 066102 (2014).

10. Handali, J., Shakya, P. \& Barwick, B. Opt. Express 23, 5236-5243 (2015).

11. Spektor, G. et al. Science 355, 1187-1191 (2017).

12. Franke-Arnold, S. et al. Opt. Express 15, 8619-8625 (2007).

\title{
Small change
}

T he 'dematerialization' of SI units so that they are defined in terms of fundamental constants is predicated on the eminently reasonable idea that this frees standards and measures from the contingent fluctuations that can occur in a reference object like the platinum-iridium standard kilogram. But what if the constants themselves are susceptible to variation? A new proposal $^{1}$ turns the tables by showing how extremely sensitive measurements on a material system could reveal such changes.

Of course, any such variation in a 'constant' of nature would have to be extremely small, or it would have been seen already. (And even if it occurs, it would not undermine a definition of units in these terms - simply, the scale would shift accordingly.) All the same, there are reasons why such inconstancy might be possible. Some theories that seek to unify the forces of nature invoke extra spatial dimensions, so that there is nothing 'fundamental' about the projection of higher-dimensional constants onto our own three dimensions: like shifting shadows, they might change both in space and over time. What is more, some theories of dark matter predict couplings to ordinary matter that can alter these constants, perhaps in an oscillatory way. Such variations might therefore offer hints about physics beyond the standard model, making them worth seeking.

One way in which they could become manifest is through a change in the sheer size of a material system.
For example, this size should change with any change in the fine-structure constant $\alpha$, which is a measure of the strength of the interaction between charged particles. The reason is subtle, arising from relativistic effects on atomic radii: the way these depend on the change of an electron's mass when it moves at an appreciable fraction of the speed of light. Another change in atomic (and thus material) dimensions could arise from variations in the ratio of the electron mass to the nuclear mass as a result of an inconstant electron/proton mass ratio $\mu$.

Pašteka et al. ${ }^{1}$ have calculated how sensitively such variations in these two constants might be probed. Ultimately the change in material dimensions arises from a shift of interatomic bond lengths (sensitive to both $\alpha$ and $\mu$ ). In principle this could be seen by looking at the dimensions of diatomic molecules, but it might be more readily studied by seeking changes in the lattice constants of inorganic crystals - in particular, using laser interferometery. If the materials are used to make optical cavities, say, then changes in dimensions would very slightly shift the resonant frequencies. For two different materials, a variation in $\alpha$ due to relativistic effects would show up as a (unit-independent) change in the ratio of these resonant frequencies. In fact, the authors say, the ratio of resonator frequency to that of an atomic clock would depend on $\alpha$ even without relativistic corrections.

Shifts in the lattice constants of elemental crystals such as copper,

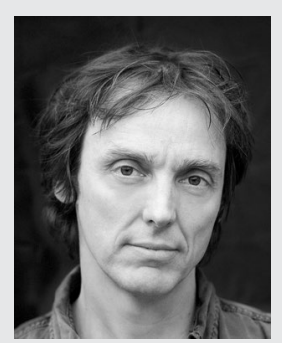

Philip Ball

silicon, aluminium, niobium and titanium, say Pašteka et al., could typically be of the order of a few thousandths of an ångström for a change in $\alpha$ of around 25\%.

That's certainly detectable, but any plausible variability of $\alpha$ would have to be a great deal smaller than that: variations in $\alpha$ have been excluded above a limit of one part in $10^{17}$ per year ${ }^{2}$. According to the new calculations, a rate of change at this limit would cause shrinkage of a gold bar by a factor of $10^{-18}$ per year. Seeing such contraction by monitoring over a few years would be a tremendous challenge - but the extreme sensitivity of laser interferometry as demonstrated by gravitational-wave detectors should make one hesitant to rule it out.

Published online: 21 May 2019 https://doi.org/10.1038/s41563-019-0390-8

References

1. Pašteka, L. F., Hao, Y., Borschevsky, A., Flambaum, V. V. \& Schwerdtfeger, P. Phys. Rev. Lett. 122, 160801 (2019).

2. Srianand, R., Chand, H., Petitjean, P. \& Aracil, B. Phys Rev. Lett. 92, 121302 (2004). 